\title{
THE EPIMORPHIC IMAGES OF A DEDEKIND DOMAIN
}

\author{
T. CHEATHAM AND E. ENOCHS
}

\begin{abstract}
We prove that the epimorphic image of a Dedekind domain $A$ is characterized (up to $A$-isomorphisms) by its torsion submodule (as an $A$-module) and the torsion-free quotient by its torsion submodule. Necessary and sufficient conditions are given on a torsion and torsion-free $A$-module in order that they be the corresponding parts of an epimorphic image of $A$.
\end{abstract}

Introduction. In this paper all rings will be associative with identity and all modules will be unitary. We require a ring homomorphism to preserve the identity. A ring homomorphism is an epimorphism if it is right cancellative in the category of rings. Let $f: A \rightarrow R$ be a ring homomorphism. If $f$ is an epimorphism and $A$ is commutative then $R$ is commutative and it is easy to see that if both $A$ and $R$ are commutative then $f$ is an epimorphism if and only if it is an epimorphism in the category of commutative rings.

The classical example of an epimorphism (which need not be surjective) is the canonical map $A \rightarrow S^{-1} A$ where $A$ is a commutative ring and $S \subset A$ is a multiplicative set. A nonclassical example is given in the introduction of [5] - let $A$ be a commutative ring, $S \subset A$ the multiplicative set generated by a nonzero element $a$ in $A$; then the "diagonal" map $A \rightarrow S^{-1} A \times A /(a)$ is an epimorphism.

It is worth noting that if $A$ is a Dedekind domain with quotient field $K$ and $\mathscr{A} \subset A$ is an ideal then, for a ring $B$ between $A$ and $K$, the diagonal map $A \rightarrow B \times A / \mathscr{Q} /$ is an epimorphism if and only if : $\mathcal{P} B=B$ for every prime ideal $\mathscr{P}$ of $A$ containing $\mathscr{C}$.

The purpose of this paper is to determine which rings $R$ can occur as the epimorphic image of a Dedekind domain $A$. If such a ring $R$ is flat as an $A$-module (equivalently, torsion-free) it is known to be a subring of the quotient field. If $R$ is torsion as an $A$-module it is a factor ring of $A$ (Corollary 1.4). If $R$ is neither torsion nor torsion-free then it is either the ring direct sum of a torsion epimorphic image and a torsion-free epimorphic image or it is a direct limit of such rings. This would seem to

Received by the editors November 12, 1971.

AMS 1969 subject classifications. Primary 1310.

Ke' words and phrases. Epimorphism, Dedekind domain, torsion submodule. 
discredit L. Silver's remark [6, p. 47] that $Z \rightarrow Q \times Z /(n)$ is a pathological example of an epimorphism.

Preliminaries. In this section $A$ and $R$ will denote rings and $f: A \rightarrow R$ a ring homomorphism. We collect some facts which will be used in proving the main theorem. We begin with a characterization of epimorphisms:

THEOREM 1. The following statements are equivalent.

(1) $f$ is an epimorphism.

(2) For any $R$-bimodule $M$ we have:

$\{x \in M \mid a x=x a$ for all $a \in A\}=\{x \in M \mid r x=x r$ for all $r \in R\}$

where we make $M$ into an A-bimodule by restriction of scalars.

(3) For each $r \in R, 1 \otimes r=r \otimes 1$ in $R \otimes_{A} R$.

(4) (resp. (4')) The canonical injection $r \mapsto r \otimes 1 \quad($ resp. $r \mapsto 1 \otimes r): R \rightarrow$ $R \otimes_{A} R$ is surjective.

(5) The canonical surjection $r \otimes r^{\prime} \mapsto r r^{\prime}: R \otimes_{A} R \rightarrow R$ is injective.

(6) (resp. $\left.\left(6^{\prime}\right)\right) R / f(A) \otimes_{A} R=(0)\left(\right.$ resp. $R \otimes_{A} R / f(A)=(0)$ ).

For all right $R$-modules $E, F$ and left $R$-module $G$ we have:

(7) $\operatorname{Hom}_{A}(E, F) \cong \operatorname{Hom}_{R}(E, F)$ (canonically).

(8) $\operatorname{Hom}_{A}(R, F) \cong \operatorname{Hom}_{R}(R, F)$.

(9) $E \otimes_{A} G \cong E \otimes_{R} G$ (canonically).

For the commutative version of this theorem see [4] and [7]. In the noncommutative case, parts of the theorem have appeared in [3] and [6] and since only minor modification of the proof for the commutative case are required we omit the proof. As an easy consequence we have:

Corollary 1.1. Suppose that $f$ is an epimorphism and $R$ is a flat right A-module (via f). Then:

(a) if $A$ has one of the following properties so does $R$-left Noetherian, left Artinian, rig'it coherent, w.gl.dim $\leqq 1$, left semihereditary, left hereditary, (von Neumann) regular;

(b) as a left A-module $R$ is an essential extension of $f(A)$.

To prove (a) one notes that a left $R$-module is $R$-injective ( $R$-flat) if and only if it is $A$-injective ( $A$-flat) and then applies part (7) or (9) of the theorem to an appropriate characterization of the class of rings under consideration. Proofs for the left Noetherian and left Artinian properties in (a) have appeared in Silver [6, Proposition 1.6, p. 48] and Popescu and Spircu [3, Corollary 2.6, p. 48].

We will prove (b) since the technique is useful.

Proof of (b). Let $r \in R, r \notin f(A)$. By Theorem 1(6), $R \otimes_{A} R / f(A)=$ (0); so in particular $1 \otimes \bar{r}=0, \bar{r}=r+f(A)$. Using the flatness of $R_{A}$, we 
know from [1, Proposition 13, p. 42] that there exist elements $x_{1}, x_{2}, \cdots$, $x_{n}$ in $R$ and elements $a_{1}, a_{2}, \cdots, a_{n}$ in $A$ such that $a_{i} \bar{r}=0 \in R / f(A)$ for all $i$, and $1=\sum_{i=1}^{n} x_{i} a_{i}$. Thus $a_{i} r \in f(A)$ for all $i$ but $a_{i} r \neq 0$ for some $i$, $1 \leqq i \leqq n$, otherwise $r=1 \cdot r=\sum x_{i} a_{i} r=0$. Therefore ${ }_{4} R$ is an essential extension of $f(A)$.

REMARK. If $A$ is an integral domain then any flat epimorphism $f: A \rightarrow R$ is an injection and so it follows from (b) that $R$ is isomorphic to a subring of the quotient field of $A$.

Corollary 1.2. Suppose $f$ is an epimorphism. If $N$ is an $R$-submodule of a right $R$-module $M$ such that $N$ is a direct summand of $M$ considered as an A-module then $N$ is a direct summand of $M$ as an $R$-module.

Proof. Trivial.

LEMMA 1.3. If $A \subset R$ is an epimorphism and $A$ is a right (or left) selfinjective ring then $A=R$.

Proof. $1_{A}$ has an extension $\sigma$ in $\operatorname{Hom}_{A}(R, R)=\operatorname{Hom}_{R}(R, R)$ whose image is $A$. Thus $\sigma(R)=A$ is an ideal in $R$ so we must have $A=R$.

Corollary 1.4. If $A$ is a Dedekind domain, $f: A \rightarrow R$ an epimorphism and $t(R)=R$ (where $t(-)$ denotes the A-torsion submodule) then for some (necessarily nonzero) ideal $\mathscr{A} \subset A, R \cong A / \mathscr{A}$.

Proof. Let $\mathscr{A}=(0: 1), 1 \in R$. Then $(0) \neq \mathscr{A}=\operatorname{Ker} f$ so $A / \mathscr{A} \cong f(A) \subset R$ is an epimorphism. But by a result of Levy and Klatt [2], $A / \mathscr{A}$ is a selfinjective ring so $A / \mathscr{A}=R$.

From now on, unless the contrary is specifically stated, $A$ will denote a Dedekind domain. A torsion $A$-module is called primary (for the prime ideal $p \subset A$ ) if each of its elements is annihilated by a suitable power of $p$. Every torsion $A$-module is uniquely a direct sum of primary modules. For an $A$-module $M, t(M)$ will denote the torsion submodule of $M$.

Lemma 1.5. Suppose $f: A \rightarrow R$ is an epimorphism. Then:

(a) each primary part of $t(R)$ is an ideal in $R$ (and hence $t(R)$ is an ideal in $R)$;

(b) $t(R)$ is reduced (i.e. has no nonzero dicisible A-submodule).

Proof. (a) Let $B$ denote the primary part of $t(R)$ for a prime $p \subset A$. Since $B$ is a pure submodule of $R, B{ }_{A} R \subset R{ }_{{ }_{1}} R$. Also, $B x_{1} R$ is $p$-primary hence $B \cdot R=\mu\left(B \otimes_{A} R\right) \subset B$.

(b) Over a Dedekind domain divisible is equivalent to injective so if $D$ denotes the maximal divisible submodule of $t(R)$ we have $D \otimes{ }_{A} R \subset$ $R \otimes_{A} R$. Since $D \otimes_{A} R$ is torsion and divisible we have $D \cdot R=$ $\mu\left(D \otimes{ }_{A} R\right) \subset D$, i.e. $D$ is an ideal in $R$. From Corollary 1.2 we see that $D$ 
is generated by an idempotent $e$ of $R$. But $(0)=D \otimes_{A} D \subset R \otimes_{A} R$ so $e=e^{2} \in D^{2}=\mu\left(D A_{A} D\right)=(0)$, i.e. $D=(0)$.

The main results.

THEOREM 2. Suppose $A$ is a Dedekind domain with quotient field $K$ and $f: A \rightarrow R$ is an epimorphism. Then $R$ is characterized, up to isomorphism, by the $A$-modules $t(R)$ and $R / t(R)$. Furthermore, $t(R)$ is a direct sum of cyclic modules $A / p^{n_{p}}$ where $p$ is a prime ideal in $A, n_{p} \in Z^{+}$and $R / t(R) \cong B$ where $A \subset B \subset K$ is a subring of $K$ for which $p B=B$ for all the primes $p$ for which the p-primary part of $t(R)$ is nonzero. Conversely, given a torsion module $T$ and a torsion-free module $F$ subject to the above conditions there is a ring $R$ and an epimorphism $A \rightarrow R$ such that $t(R) \cong T$ and $R / t(R) \cong F$.

Proof. If $t(R)=(0), R$ is a flat $A$-module and by a previous remark $R$ is isomorphic to a subring of the quotient field $K$ of $A$. It is well known that any subring of $K$ containing $A$ is uniquely determined by the prime ideals $p$ of $A$ such that $p B=B$. Assume that $t(R) \neq(0)$. Then for some prime ideal $p$ of $A, t(R)$ has a nonzero $p$-primary part $P$. Since $P$ is a pure submodule of $R$ (i.e. $a P=a R \cap P$ for all $a \in A$ ), the homomorphism $P / p P \rightarrow R / p R$ is an injection but $P$ is reduced by Lemma 1.5 so $P \neq p P$. Hence $R / p R \neq(0)$. But the induced ring homomorphism $\bar{f}: A / p \rightarrow R / p R$ is $1-1$ and an epimorphism hence a surjection (Lemma 1.3). Thus $A / p \cong R / p R \cong P / p P$ (as $A$-modules).

Now any reduced $A$-module which is not torsion-free has a cyclic direct summand-so write $P=G \oplus C$ where $(0) \neq C$ is a cyclic $A$-module. Then $p C \neq C$ so $P / p P \cong G / p G \oplus C / p C$ implies $G / p G=(0)$, i.e. $G=p G$ is a divisible submodule of $P$, hence $G=(0)$. It follows that $P=C$ is a direct summand of $R$ (as an $A$-module). By Corollary 1.2, $P$ is generated by an idempotent of $R$. Therefore, if $t(R)$ has only a finite number of nonzero primary components, $t(R)$ is generated by an idempotent of $R$. Then the composition $A \rightarrow{ }^{f} R \rightarrow{ }^{\mathrm{proj}} t(R)$ is an epimorphism so, by Corollary $1.4, t(R) \cong$ $A / \mathscr{A}\left(\cong A / p_{1}^{c_{1}} \cdots \cdot A / p_{n}^{e_{n}}\right)$ for an appropriate ideal $\mathscr{A} \neq(0)$ of $A$. Also, $R / t(R)$ is a flat epimorphic image of $A$ so isomorphic to $B$ for some subring $A \subset B \subset K$. If the $p$-primary part of $t(R)$ is nonzero then $p t(R) \neq t(R)$ and since $R / p R \simeq t(R) / p t(R) \oplus B / p B$ is simple we have $p B=B$.

Now assume that $t(R)$ is infinite-say $t(R)=\oplus_{p \in X} A / p^{n_{p}}, n_{p} \geqq 1, X$ an infinite set of prime ideals in $A$. Let $X^{\prime}$ be a finite subset of $X$ and $e$ an idempotent in $R$ generating $\oplus_{p \in X^{\prime}} A / p^{n_{p}}$. If $q$ is a prime not in $X$, every element of $t(R)$ is uniquely divisible by elements of $q$. So if an element of $q$ is invertible in $R / t(R)$ it is invertible in $R$ so in $R(1-e)$. Also, if $q \in X^{\prime}$, $q R e \neq R e$ and since $R / q R \cong \operatorname{Re} / q \operatorname{Re} \oplus R(1-e) / q R(1-e)$ is a simple $A$ module we must have $q R(1-e)=R(1-e)$. Hence some element of $q$, for $q \in X^{\prime}$, is invertible in $R(1-e)$. 
Now for any such finite subset $X^{\prime} \subseteq X$, let $R_{X^{\prime}}$ denote the subring of $R$ generated by $R e=\oplus_{p \in X^{\prime}} A / p^{n_{p}}$ and all $\alpha^{-1} \in R(1-e)$ such that $\alpha \in A$ and $\alpha^{-1} \in R / t(R)$ or $\alpha \in q$ with $q \in X^{\prime}$.

The following facts are easy to check: $R_{X}$, is an epimorphic image of $A ; t\left(R_{X^{\prime}}\right)=R e ; R$ is the direct limit of the rings $R_{X^{\prime}}, X^{\prime}$ a finite subset of $X$.

To see that $R$ is uniquely determined by the modules $t(R)=\oplus_{p \in X} A / p^{n_{p}}$ and $F \cong R / t(R)$ let $A \rightarrow R^{*}$ be another epimorphism such that $t(R) \cong t\left(R^{*}\right)$ and $R^{*} / t\left(R^{*}\right) \cong F$. From above $t\left(R^{*}\right) \cong \oplus_{p \in X^{*}} A / p^{n_{p} p^{*}}$, and it follows that $X=X^{*}, n_{p}=n_{p}^{*}$.

For each finite subset $X^{\prime} \subset X=X^{*}$ we have a ring homomorphism $R_{X^{\prime}} \rightarrow R_{X^{\prime}}^{*}$ (where the rings $R_{X^{\prime}}, R_{X^{\prime}}^{*}$ are constructed as above) which is an isomorphism and these isomorphisms are compatible with the directed systems $\left\{R_{X^{\prime}} \rightarrow R_{X^{\prime \prime}}\right\},\left\{R_{X^{\prime}}^{*} \rightarrow R_{X^{\prime \prime}}^{*}\right\}$ so we have an isomorphism

$$
R=\operatorname{inj} \lim R_{X^{\prime}} \rightarrow \operatorname{inj} \lim R_{X^{\prime}}^{*}=R^{*} .
$$

Now suppose we are given a torsion module $T=\oplus_{p \in X^{\prime}} A / p^{n_{p}}$ where $X$ is a set of prime ideals in $A, n_{p} \geqq 1$ for all $p \in X$, and an $F \cong B$ where $p B=B$ for all $p \in X$. We want to construct $R$ with these invariants. Let $Y$ denote the set of prime $q$ in $A$ for which $q B=B$. Let $R$ denote the subring of $\prod_{p \in X} A / p^{n_{p}}$ generated by $\oplus_{p \in X} A / p^{n_{p}}$ and the following sets:

$G_{1}=\left\{\left(x_{p}\right) \in \prod A / p^{n_{p}} \mid\right.$ there exist $q \in X$ and $\alpha \in q$ with

$$
\left.x_{p}=\alpha^{-1} \in A / p^{n_{p}} \text { if } p \neq q \text { and } x_{q}=0\right\} \text {, }
$$

$G_{2}=\left\{\left(x_{p}\right) \in \prod A\left|p^{n_{p}}\right|\right.$ there exist $q \in Y, q \in X$, and $\alpha \in q$ with

$$
\left.x_{p}=\alpha^{-1} \in A / p^{n} \text { for all } p \in X\right\} \text {, }
$$

One easily checks that $t(R)=\oplus_{p \in X} A / p^{n_{p}}$ is an ideal in $R$ and if $p \in Y$. $p R / t(R)=R / t(R)$. Hence there is a ring injection $B \rightarrow R / t(R)$. Since $R / t(R)$ is generated by the images of elements in $G_{1} \cup G_{2}$ we have $B \cong R / t(R)$.

Finally, we can show that $R$ is an epimorphic image of $A$ by realizing $R$ as the direct limit of its subrings $R_{X^{\prime}}$, where $X^{\prime}$ is a finite subset of $X$ and $R_{X^{-}}$is constructed as before.

\section{REFERENCES}

1. N. Bourbaki, Éléments de mathématique. Fasc. XXVII. Algèbre commutative. Chaps. 1, 2, Actualités Sci. Indust., no. 1290, Hermann, Paris, 1961. MR 36 \#146.

2. L. S. Levy and G. B. Klatt, Pre-self-injective rings, Trans. Amer. Math. Soc. 137 (1969), 407-419. MR 38 \#4463.

3. N. Popescu and T. Spircu, Quelques observations sur les épimorphisms plat (à gauche) d'anneaux, J. Algebra 16 (1970), 40-59. MR 42 \#323. 
4. N. Roby, Sur les épimorphismes de la catégorie des anneaux, C. R. Acad. Sci. Paris Sér. A-B 266 (1968), A312-A313. MR 39 \#197b.

5. P. Samuel, Séminaire d'algèbre commutative dirigé par Pierre Samuel: 1967/68. Les épimorphismes d'anneaux, Secrétariat mathématique, Paris, 1968. MR 39 \#6867.

6. L. Silver, Noncommutative localizations and applications, J. Algebra 7 (1967), 44-76. MR 36 \#205.

7. H. Storrer, Epimorphismen von kommutativen Ringen, Comment. Math. Helv. 43 (1968), 378-401. MR 39 \#4137.

Department of Mathematics, Samford University, Birmingham, Alabama 35209

Department of Mathematics, University of Kentucky, Lexington, Kentucky 40506 